\title{
Unintended Return of Consciousness in a Patient during Surgery and General Anesthesia
}

\author{
Wioletta Mędrzycka-Dąbrowska ${ }^{a}$ Sebastian Dąbrowski ${ }^{b} \quad$ Aleksandra Gutysz-Wojnickac $^{c}$ \\ Dorota Ozga $^{d}$ Marek Wojtaszek $^{d}$ \\ ${ }^{a}$ Department of General Nursing, Medical University of Gdańsk, and ${ }^{b}$ Department of Anesthesiology and \\ Intensive Care, Specialistic Hospital, Gdańsk, 'Department of Nursing, Faculty of Medical Sciences, University of \\ Warmia and Mazury in Olsztyn, Olsztyn, and ${ }^{\mathrm{d} D e p a r t m e n t}$ of Emergency Medicine, Faculty of Medicine, \\ University of Rzeszow, Rzeszow, Poland
}

Dear Sir,

The term "awareness" during anesthesia, as used in the anesthesia literature, means that during a period of intended general anesthesia, the brain is aroused by stimuli that are stored in memory for future explicit recall [1]. Patients who experience awareness will recall such experiences during a state of inadequate anesthesia. Although it is an uncommon phenomenon - occurring in about $0.1-0.2 \%$ of cases - it is receiving increased attention from patients, clinicians, and researchers worldwide. Awareness may lead to postoperative sequelae that may persist for varying durations. Late psychological symptoms may lead to a severe and debilitating illness - post-traumatic stress disorder (PTSD) $[2,3]$.

"In 1998 Carol Weiher of Reston, Virginia was undergoing a surgical removal of her right eye, at some point she became fully aware being able to hear disco music. She also managed to hear 'cut deeper, pull harder.' She was not able to scream or move her finger to communicate with doctors because the muscle relaxant she had been administered prevented her from doing any controlled movement. She stated later that she silently prayed, pleaded, cursed and screamed trying to do anything she could but in vain" [4].

The Joint Commission on Accreditation of Hospital Organizations (JCAHO), which is an independent and non-profit organization accrediting and certifying health care organizations and programs all around the United States, decided to publish Sentinel Alert in 2004 to give more attention to this issue $[2,5]$.

\section{Aim}

The aim of the study was to analyze factors associated with the return of consciousness during general anesthesia.

\section{Material and Methods}

We searched PubMed/Medline databases for records including the following phrases "awareness under general anesthesia," "BIS monitoring," "BIS monitoring prevents awareness under general anesthesia," "prevention of awareness during general anesthesia," and "general anesthesia complications." The search was limited only to human studies in English and Polish from last 12 years (2004-2016). While searching we typed single keywords, their combinations, and used Boolean operators AND, OR, or both. The number of citations acquired in each search was scanned and reduced using inclusion criteria, which covered surgical patients experi- encing awareness under general anesthesia. The reduction process began with a perusal of titles cited. After this process of filtering, abstracts were obtained. The acquired abstracts were then read; their numbers were reduced to selected relevant articles. References, or bibliographies, of these articles were accessed to expand the original searches. Abstracts of these citations were filtered for relevant information. The final list of references was based on relevance to the topics covered, which included prevalence, detection, and solutions for prevention of awareness under general anesthesia.

\section{General Anesthesia}

During general anesthesia, distinct neural functions are dynamically suppressed to varying degrees. General anesthetics affect a wide variety of neural functions, suppressing different neural circuits at different concentrations. Although there is no established consensus defining general anesthesia, the most important therapeutic actions of all general anesthetics include suppression of memory, awareness (consciousness), and movement. Suppression of these 3 central nervous system (CNS) functions occurs at different anesthetic concentrations. In addition, each of these anesthetic-sensitive actions is controlled by different neural circuits in the CNS,

\section{KARGER}

(c) 2017 S. Karger AG, Basel 
which are also anatomically distinct. Different general anesthetics also act via distinct mechanisms at the molecular level. Opioids given by themselves have relatively weak hypnotic effects, although large doses of opioids will produce unconsciousness [6-9]. As a consequence, opioids do not alter the incidence of awareness when added to nitrous oxide. When opioids are combined with typical hypnotic drugs, such as propofol, there is a synergistic effect that dramatically strengthens the anesthetic effect of the combination of drugs. Anesthetic regimens containing opioids are likely to produce less-responsive patients at any given monitoring index value, compared with anesthetic regimens containing no opioids or lesser amounts of opioids. Propofol, barbiturates, etomidate, and halogenated volatile anesthetic agents all modulate the $\mathrm{GABA}_{\mathrm{A}}$ receptor activity and shift the cortical EEG to lower frequencies. Nitrous oxide and ketamine (xenon and cyclopropane, too) do not modulate $\mathrm{GABA}_{\mathrm{A}}$ receptors, but they do produce hypnosis [9-12].

\section{Intraoperative Awareness - Causes and Risk Factors}

The causes of intraoperative awareness are not yet clear, and it seems to be multifactorial problem. As many as 4 types of plausible causes can be distinguished. First, unanticipated patient-specific variance in required dose of anesthetic results from changed expression or function of target receptors. Second, patients may not tolerate a precalculated anesthetic dose due to low physiological reserves connected with such factors as cardiac dysfunction or lack of euvolemia. Third, physiological characteristics that would imply the need for a changed dose may be masked by such factors as the presence of a pacemaker or use of $\beta$-adrenergic blockade. Fourth, intended systems of drug delivery may be compromised due to such events as apparatus failure or mismanagement. The first category might prove particularly disturbing since the patient would be subjected to a dose of an anesthetic, which seems adequate; however, the depth of anesthesia obtained would be inadequate [10-15]. The factors that influence the risk of awareness during general anesthesia include those associated with the anesthetic technique, the type of surgery, and the patient. A critical factor is the use of neuromuscular blockade. When muscle relaxants are not used during general anesthesia, patients can move, and the absence of movement suggests that the patient is adequately anesthetized, or if aware, not suffering. Another significant risk is "light" anesthesia techniques, particularly cardiac anesthesia without hypnotic agents and $\mathrm{N}_{2} \mathrm{O}$-narcotic-relaxant, which has been associated with up to $4 \%$ awareness. It is therefore expected that the highest risk surgeries are those associated with light anesthesia, which is often intentionally used to prevent the hemodynamic consequences of high concentrations of volatile or intravenous agents. These include cardiac surgery (1-1.5\% awareness), trauma surgery (11-43\% awareness), and Cesarean section under general anesthesia (0.4\%), where light anesthesia is used to minimize newborn depression. Patient-related factors include chronic alcohol, anti-epileptic, opiate, or other sedative drug use (associated with higher anesthetic requirements), history of awareness during general anesthesia, limited cardiovascular reserve, or ASA class IV-V (low tolerance for adequate anesthesia) [16-18].

\section{Monitoring and Detection}

Awareness occurs partly as a result of the lack of ability to precisely evaluate the depth of anesthesia, especially when it comes to the awareness-blocking part of anesthesia. The anesthetic dose is best modified by a knowledgeable anesthesia provider, who relies on numerous parameters to assess the depth of anesthesia, such as heart rate patterns, blood pressure, lacrimation, and movement. Typical intraoperative monitoring includes pulse oximetry; noninvasive blood pressure; electrocardiogram tracings; airway pressure; and concentration of oxygen, carbon dioxide, and other anesthetic gases $[19,20]$. Unfortunately, no variations in hemodynamic parameters occurred during episodes of awareness. Clinical signs monitoring is supplemented by monitors designed to assess the electrical activity in the brain, while the patient is under the influence of anesthesia. Two general categories of such monitoring systems can be distinguished: those intended to analyze electroencephalographic activity with electrodes placed on the patient's forehead and the other detect and analyze evoked responses to auditory stimuli. There are devices that also analyze electromyographic activity of scalp muscles. The electrical activity measured by them does not directly present the depth of sleep or memory in a quantifiable way, but it may show correlated neuronal activities. Additionally, the sensitivity of measurements with these devices is not uniform and varies among different anesthetic drugs, their combinations or patients' types. The first product available for routine intraoperative EEG monitoring of anesthetic depth was produced by Aspect Medical Systems (Newton, Massachusetts) and given the name BIS from bispectral analysis, which is part of the algorithm used to interpret the EEG. The US Food and Drug Administration in 1996 approved the BIS sensor. By 2001, 4 million patients had been monitored. BIS is by far the most extensively used and studied depth-of-anesthesia monitor. The cortical EEG of a normal, awake subject is characterized by fast, low-amplitude activity. The administration of most, but not all, anesthetic drugs initially results in increased amplitude, followed at larger doses by decreased frequency and increased regularity, and finally, at very deep levels, periods of isoelectric (flat) EEG interspersed with bursts of undulating EEG activity (burst suppression). The BIS algorithm (or similar algorithms for other EEG-based depth-ofanesthesia monitors) uses a statistical process to analyze the EEG and compute an index on a linear scale from 0 to 100 , where 0 is an isoelectric EEG. The algorithm is empirical and is not based upon a physical law or a simple equation. The main variables incorporated in the algorithm are the frequency and power spectrum of the EEG, the amount of burst suppression, and the degree of synchronization of the EEG [21,22]. The BIS index is a number from 0 to 100 . A variety of clinical studies were performed to validate the index and define the relationship to clinical end-points. The probability of explicit recall decreases dramatically below a BIS index of 70 , and below 60 , the probability of explicit recall becomes extremely small. At BIS index values below 40 , the index is determined primarily by the degree of "suppression" or isoelectricity of the EEG. The BIS index values correlate to the reduction in cerebral metabolic rate measured from positron emission tomographic scanning during administration of hypnotic drugs. Numerous studies have shown that the use of the BIS index to guide the administration of anesthetic drug results in administration, on average, of less anesthetic drug $[23,24]$.

What is noteworthy about intraoperative awareness is that it may take place even if monitoring devices are on - since there are no flawless monitoring devices, and there should be no surprise if depth-of-an- 
esthesia monitoring fails to perform in some cases. Obviously failures have to be kept to a minimum for monitoring to be useful. The worst kind of failure for a depthof-anesthesia monitor would be intraoperative awareness that occurs despite an index value in the recommended range for general anesthesia (e.g., <60 for BIS). Index ranges are established based on the probability of finding a particular state (e.g., awake versus asleep) within a particular range of index values. In other words, the probability of awareness will be very low if the patient has an index below a certain level, but the probability will not be zero, and awareness would be expected to occur occasionally with an index value within the recommended range. However, the actual failure rate for the recommended ranges in a large population is not known with certainty for any of the monitors. For the BIS monitor, only 2 prospective studies show that BIS monitoring is effective for reducing the incidence of intraoperative awareness compared with no depth-of-anesthesia monitoring, which suggests that the recommended index range for general anesthesia for BIS probably has a very low failure rate. Case reports are important for providing additional, albeit anecdotal, information about monitoring failures. A cluster of reports of intraoperative awareness that occurs with index values within the recommended range would certainly suggest a problem with the recommended range. Interestingly, case reports of intraoperative awareness that occur with index values in the recommended range have been rare. Mychaskiw reported a case of intraoperative awareness during sternotomy apparently at a BIS index value of 47 , based on review of the BIS values recorded on the anesthesia record. However, subsequent to the publication of the case report, analysis of the flash memory of the monitor (BIS monitors have a flash memory that stores around 30 days of cases under typical conditions of use) revealed BIS index values $>60$ occurred at the approximate time of the awareness episode and were not recorded on the anesthesia record. This additional information was published in a subsequent report. Rampersad and Mulroy subsequently published another report of intraoperative awareness with BIS index values $<60$ recorded every $15 \mathrm{~min}$ on the anesthesia record. The flash memory was apparently not accessed for additional data. In addition to these reports, there is one case of intraoperative awareness with a BIS index value $<60$ during cardiac surgery contained in the multicenter prospective randomized trial of BIS monitoring for the prevention of intraoperative awareness. There may be other case reports that have not come to the attention of this author. However, considering the large number of patients who have been monitored, the number of case reports remains remarkably small. Cases of intraoperative awareness that occur during depth-of-anesthesia monitoring with index values within the recommended range should be reported in the peer-reviewed literature. If possible, the memory of the monitor should be accessed to be sure that the index values recorded in the anesthetic record are indicative of the range of index values that actually occurred during the episode of intraoperative awareness [19, 24]. Most clinical cases of awareness during general anesthesia are elicited using a postanesthesia structured interview, which is sometimes repeated at intervals after surgery. For a variety of reasons, patients rarely report such experiences to their anesthetists without prompting, so it is critical that anesthetists habitually perform postoperative interviews that incorporate specific questions designed to elicit reports of awareness. Intraoperative awareness can be characterized by duration, the experience of pain and/or anxiety, and whether explicit recall is present. Without question, the most disturbing cases are those of prolonged "awake paralysis," where patients are aware, experiencing pain and anxiety, and able to remember these experiences. It appears that most cases of awareness with explicit recall are brief, and usually no pain is experienced. In addition, a large number of cases fall into the category of awareness without explicit recall. In these, patients report only vague memories, which may or may not be associated with intraoperative events. Finally, some case series include dreams or dream-like experiences, which have been judged to be associated with intraoperative events [25].

\section{Psychological Consequences}

After experiencing intraoperative awareness, some patients have developed PTSD, which is considered the most harmful consequence. Symptoms include depression, anxiety attacks, sleep disorders, flashbacks to the experience, and nightmares. Although rare, PTSD has also been diagnosed in patients who have no explicit recall of intra-operative events, but who develop symptoms suggestive of intraopera- tive awareness, such as recurrent dreams about being buried alive or burying friends or family members alive. A patient's understanding of their experiences can affect the psychological impact of awareness during general anesthesia. Patients may think that it is impossible to become conscious while they are under general anesthesia (uninformed healthcare workers may also deny this possibility), leading them to become confused or question their own sanity. In a number of cases, when a patient's experience is validated and explained by a physician, their symptoms of PTSD diminish or stop. In others, patients remain "victims" for prolonged periods of time [25-28]. Compared to the estimates of awareness during the general anesthesia incidence, we know even less about the incidence of PTSD. A follow-up to Sandin's Swedish incidence study by Lennmarken et al. suggests that half or more of the patients who experienced awareness with recall developed long-term psychological problems despite having had 3 weeks of contact and repeated offers of psychological support from the research team immediately after their surgery [29]. It remains controversial whether repeated debriefing is helpful or harmful to people following traumatic experiences. There are data suggesting that PTSD is worsened when recall of traumatic events is associated with a stress response (and thus repeated debriefing may worsen things). One preliminary report suggests that PTSD may be attenuated by the early administration of beta-blockers, effectively uncoupling memory from the stress response. A small fraction of patients who experience awareness during general anesthesia initiate legal action against their anesthesia providers. Most of these are women who also disproportionately complain of PTSD symptoms [26]. In the ASA closed claims database from 1971 to 2001, the percentage of claims against anesthetists for intraoperative awareness grew from 1 to $3 \%$, and it is likely to continue growing. Cases of intraoperative awareness, especially with explicit recall, are difficult to defend, because it is assumed that general anesthesia is supposed to make patients unconscious. As of 2001, reported awards to patients for awareness with recall ranged from $\$ 1,000$ to $\$ 600,000$, but the median award for psychological damages from intraoperative awareness was small $(\$ 18,000)$ in comparison to that for other anesthesia-related injuries $(\$ 100,000)$. Should the patient experience or claim to have had awareness during gen- 
eral anesthesia, there are several actions that can potentially reduce the psychological harm to the patient and the probability of legal action against caregivers.

When a patient reports awareness during general anesthesia [27-29]:

- Get as much information about patient's experience as possible. Record the detailed account in their chart.

- Verify patient's story.

- Acknowledge your patients' stressful experiences and be sympathetic towards them. Explain what happened to the patients and answer all of their questions.

- Inform the surgeon(s) and nurse(s) involved in the case and a hospital lawyer.

- If patients experiences symptoms of PTSD, refer them to a knowledgeable psychiatrist.

\section{Prevention}

There are suggested a number of preventive measures to help avoid intraoperative awareness. Prevention of awareness during general anesthesia and its consequences - suggestions for anesthesia providers [30-34]:

- Check equipment carefully before use.

- Premedicate with amnestic. They synergize with anesthetics to prevent awareness. Also, if awareness occurs, psychological trauma is less likely without recall.

- Avoid muscle relaxants and minimize their use when needed.

\section{References}

1 Chung HS: Awareness and recall during general anesthesia. Korean J Anesthesiol 2014;66: 339-345.

2 Aranake A, Gradwohl S, Ben-Abdallah A, et al: Increased risk of intraoperative awareness in patients with a history of awareness. Anesthesiology 2013;119:1275-1283.

3 Mohamed M, Ghoneim MD: Awareness during anesthesia: risk factors, causes and sequelae: a review of reported cases in the literature. Anesth Analg 2009;108:527-535.

$4 \mathrm{http}: / /$ edition.cnn.com/2010/ HEALTH/05/17/general.anesthesia/.

5 Sebel PS, Bowdle TA, Ghoneim MM, Rampil IJ, Padilla RE, Gan TJ, Domino KB: The incidence of awareness during anesthesia: a multicenter United States study. Anesth Analg 2004;99:833-839.

6 Orser BA, Mazer CD, Baker AJ: Awareness during anesthesia. CMAJ 2008;178:185-188.

7 Lopez U, Habre W, Laurençon M, Haller G, Van der Linden M, Iselin-Chaves IA: Intra-
- Avoid light induction doses. Re-bolus with intravenous hypnotic during multiple intubation attempts. Consider using the inhalation-induction technique.

- Supplement $\mathrm{N}_{2} \mathrm{O}$ /narcotic with volatile or propofol, if possible.

- Assure agent delivery using End-tidal gas monitors. Check drug infusion pumps frequently.

- Consider beta-blockers, which may reduce MAC-Awake and may also decrease the likelihood of PTSD.

- Consider earplugs or headphones to reduce patient awareness of noises in the OR.

- Inform high-risk patients about awareness during general anesthesia, and consider doing so for all patients.

- Routinely ask your post-op patients questions designed to detect awareness.

- Educate yourself about awareness during general anesthesia.

- Consider EEG-based monitoring in high-risk cases.

\section{Conclusion}

Despite the ongoing research, there seem to be many unresolved and unclear issues surrounding the topic of explicit intraoperative recall, for instance:

- There is still no ideal depth of anesthesia monitoring equipment.

- There are no specific human studies on perioperative drugs and increase in in-

operative awareness in children: the value of an interview adapted to their cognitive abilities. Anaesthesia 2007;62:778-789.

8 Sandin RH, Enlund G, Samuelsson P: Awareness during anaesthesia: a prospective case study. Lancet 2000;355:707-711.

9 Stuart A, Forman MD: Awareness during general anesthesia: concepts and controversies. Semin Anesth Perioperat Med Pain 2006; 25:159-171.

10 Pollard RJ: Intraoperative awareness in a regional medical system: a review of 3 years' data. Anesthesiology 2007;106:269-274.

11 Muzolf J, Onichimowski D, Podlińska I: Unintentional return of consciousness during general anaesthesia (awareness). Anestezjologia i Ratownictwo 2008;2:314-319.

12 Shosholcheva M, Jankulovski N, Kuzmanovska B, Kartalov A: Incidence of anesthetic awareness may be higher in low flow anesthesia. J Anesth Crit Care Open Access 2016;4: 00147. traoperative awareness, though there is some animal data suggesting such a link.

- An issue of relationship between the depth of anesthesia and adverse outcomes needs to be clarified.

Anesthesia providers should educate themselves thoroughly about intraoperative awareness and pre-anesthetic evaluation should routinely include the assessment of patients' risk for this problem [3538]. Strategies to reduce the chance of awareness should be applied whenever possible, unless these increase the risk of other unfavorable outcomes. Postoperatively, patients should be asked questions designed to elicit reports of awareness experiences [39, 40]. When intraoperative awareness is suspected, the responsible anesthesia providers, their departmental administrators, and quality assurance team members should activate a series of interventions aimed at defining the nature of the event and its causes, while minimizing its impact by providing supportive care to patients. Patients assessed to be at high risk for intraoperative awareness should be informed about their status and anesthetic plans should explicitly incorporate approaches to reduce this risk, including the use of EEG-based monitoring when feasible [32].

\section{Disclosure Statement}

The authors report no conflicts of interest.

13 Mashour GA, Avidan MS: Intraoperative awareness: controversies and non-controversies. Br J Anaesth 2015;115(suppl 1):i20i26.

14 Cascella M, Schiavone V, Muzio MR, Cuomo A: Consciousness fluctuation during general anesthesia: a theoretical approach to anesthesia awareness and memory modulation. Curr Med Res Opin 2016;32:1351-1359.

15 Cook TM, Andrade J, Bogod DG, et al: 5th National Audit Project (NAP5) on accidental awareness during general anaesthesia: patient experiences, human factors, sedation, consent, and medicolegal issues. Br J Anaesth 2014;113:560-574.

16 Bowdle TA: Depth of anesthesia monitoring. Anesthesiol Clin 2006;24:793-822.

$17 \mathrm{Xu} \mathrm{L}, \mathrm{Wu}$ AS, Yue Y: The incidence of intraoperative awareness during general anesthesia in China: a multi-center observational study. Acta Anaesthesiol Scand 2009;53:873882 . 
18 Sandhu K, Dash H: Awareness during anaesthesia. Indian J Anaesth 2009;53:148-157.

19 Schneider G, Gelb AW, Schmeller B, Tschakert R, Kochs E: Detection of awareness in surgical patients with EEG-based indices bispectral index and patient state index. $\mathrm{Br}$ Anaesth 2003;91:329-335.

20 Kerssens C, Gaither JR, Sebel PS: Preserved memory function during bispectral indexguided anesthesia with sevoflurane for major orthopedic surgery. Anesthesiology 2009;111: 518-524.

21 Soehle M, Kuech M, Grube M, et al: Patient state index vs bispectral index as measures of the electroencephalographic effects of propofol. Br J Anaesth 2010;105:172-178.

22 Myles PS, Leslie K, McNeil J: Bispectral index monitoring to prevent awareness during anaesthesia: the B-Aware randomised controlled trial. Lancet 2004;363:1757-1763.

23 Bischoff P, Rundshagen I: Awareness under general anesthesia. Dtsch Arztebl Int 2011 108:1-7.

24 Zhang C, Xu L, Ma YQ, et al: Bispectral index monitoring prevent awareness during total intravenous anesthesia: a prospective, randomized, double-blinded, multi-center controlled trial. Chin Med J (Engl) 2011;124 3664-3669.

25 Radovanovic D, Radovanovic Z: Awareness during general anaesthesia - implications of explicit intraoperative recall. Eur Rev Med Pharmacol Sci 2011;15:1085-1089.

26 Samuelsson P, Brudin L, Sandin RH: Late psychological symptoms after awareness among consecutively included surgical patients. Anesthesiology 2007;106:26-32.

27 Avidan MS, Jacobsohn E, Glick D, et al: Prevention of intraoperative awareness in a highrisk surgical population. N Engl J Med 2011; 365:591-600.

28 Mashour GA, Shanks A, Tremper KK, et al: Prevention of intraoperative awareness with explicit recall in an unselected surgical population: a randomized comparative effectiveness trial. Anesthesiology 2012;117:717725.

29 Lennmarken C, Bildfors K, Enlund G, et al: Victims of awareness. Acta Anaesthesio Scand 2002;46:229-231.

30 American Society of Anesthesiologists Task Force on Intraoperative Awareness: Practice advisory for intraoperative awareness and brain function monitoring: a report by the American society of anesthesiologists task force on intraoperative awareness. Anesthesiology 2006;104:847-864.

31 Ziętkiewicz M, Nestorowicz A: Intraoperative awareness - recommendations of the committee on quality and safety in anaesthesia, polish society of anaesthesiology and intensive therapy. Anaesthesiol Intensive Ther 2012;44:57-62.

32 Morimoto Y, Nogami Y, Harada K, et al: Awareness during anesthesia: the results of a questionnaire survey in Japan. J Anesth 2011; 25:72-77.

33 Pandit JJ, Cook TM, Jonker WR, et al: A national survey of anaesthetists (NAP5 baseline) to estimate an annual incidence of ac- cidental awareness during general anaesthesia in the UK. Br J Anaesth 2013;110:501-509.

34 Pandit JJ, Andrade J, Bogod DG, et al: 5th National Audit Project (NAP5) on accidental awareness during general anaesthesia: summary of main findings and risk factors. Br J Anaesth 2014;113:549-559.

35 Kerssens C and Sebel PS: Intraoperative awareness and recall; in Lobato EB (ed): Complications in Anesthesiology. Philadelphia, Lippincott Williams \& Wilkins, 2008, pp 365-375.

36 Szostakiewicz KM, Tomaszewski D, Rybicki Z, Rychlik A: Intraoperative awareness during general anaesthesia: results of the observational survey. Anaesthesiol Intensive Ther 2014;46:23-28

37 Errando CL, Sigl JC, Robles M, et al: Awareness with recall during general anaesthesia: a prospective observational evaluation of 4001 patients. Br J Anaesth 2008;101:178-185.

38 Wacker J, Staender S: The role of the anesthesiologist in perioperative patient safety. Curr Opin Anaesthesiol 2014;27:649-656.

39 Hasak L, Wujtewicz M, Owczuk R: Assessment of the depth of anaesthesia during inhalational and intravenous induction of general anaesthesia. Anaesthesiol Intensive Ther 2014;46:274-279.

40 Czarko K, Kwiatosz-Muc M, Fijałkowska A, Kowalczyk M, Rutyna R: Intraoperative awareness - comparison of its incidence in women undergoing general anaesthesia for Caesarean section and for gynaecological procedures. Anaesthesiol Intensive Ther 2013;45:200-204. 